\title{
Effects of 12-week cadence retraining on impact peak, load rates and lower extremity biomechanics in running
}

\author{
Junqing Wang ${ }^{1}$, Zhen Luo ${ }^{1}$, Boyi Dai ${ }^{2}$, Weijie Fu ${ }^{\text {Corresp. } 1,3}$ \\ ${ }^{1}$ School of Kinesiology, Shanghai University of Sport, Shanghai, China \\ 2 Division of Kinesiology and Health, University of Wyoming, Laramie, United States of America \\ 3 Key Laboratory of Exercise and Health Sciences of Ministry of Education, Shanghai University of Sport, Shanghai, China \\ Corresponding Author: Weijie Fu \\ Email address: fuweijie@sus.edu.cn
}

Background Excessive impact peak forces and vertical load rates are associated with running injuries and have been targeted in gait retraining studies. This study aimed to determine the effects of 12-week cadence retraining on impact peak, vertical load rates and lower extremity biomechanics during running. Methods Twenty-four healthy male recreational runners were randomised into either a 12-week cadence retraining group ( $n=$ 12 ), which included those who ran with a $7.5 \%$ increase in preferred cadence, or a control group, which included those who ran without any changes in cadence $(n=12)$. Kinematics and ground reaction forces were recorded simultaneously to quantify impact force variables and lower extremity kinematics and kinetics. Results Significantly decreased impact peak ( $1.86 \pm 0.30 \mathrm{BW}$ vs. $1.67 \pm 0.27 \mathrm{BW}, P=0.003)$, vertical average load rates (91.59 $\pm 18.91 \mathrm{BW} / \mathrm{s}$ vs. $77.31 \pm 15.12 \mathrm{BW} / \mathrm{s}, P=0.001)$ and vertical instantaneous load rates $(108.8 \pm 24.5 \mathrm{BW} / \mathrm{s}$ vs. $92.8 \pm 18.5 \mathrm{BW} / \mathrm{s}, P=0.001)$ were observed in the cadence retraining group, while no significant differences were observed in the control group. Foot angles $\left(18.27^{\circ} \pm 5.59^{\circ}\right.$ vs. $\left.13.74^{\circ} \pm 2.82^{\circ}, P=0.003\right)$ and vertical velocities of the centre of gravity $(\mathrm{CoG})(0.706 \pm 0.115 \mathrm{~m} / \mathrm{s}$ vs. $0.652 \pm 0.091 \mathrm{~m} / \mathrm{s}, P=0.002)$ significantly decreased in the cadence retraining group at initial contact, but not in the control group. In addition, vertical excursions of the CoG $(0.077 \pm 0.01 \mathrm{~m}$ vs. $0.069 \pm 0.008 \mathrm{~m}, P=0.002)$ and peak knee flexion angles $\left(38.6^{\circ} \pm 5.0^{\circ}\right.$ vs. $\left.36.5^{\circ} \pm 5.5^{\circ}, P<0.001\right)$ significantly decreased whilst lower extremity stiffness significantly increased (34.34 $\pm 7.08 \mathrm{kN} / \mathrm{m}$ vs. $38.61 \pm 6.51 \mathrm{kN} / \mathrm{m}, P=0.048$ ) in the cadence retraining group. However, no significant differences were observed for those variables in the control group. Conclusion Twelveweek cadence retraining significantly increased the cadence of the cadence retraining group by $5.7 \%$. This increased cadence effectively reduced impact peak and vertical average/instantaneous load rates. Given the close relationship between impact force variables and running injuries, increasing the cadence as a retraining method may 
potentially reduce the risk of impact-related running injuries. 


\section{Effects of 12-week cadence retraining on impact peak, load \\ 2 rates and lower extremity biomechanics in running}

4 Junqing Wang ${ }^{1}$, Zhen $\mathrm{Luo}^{1}$, Boyi $\mathrm{Dai}^{2}$, Weijie $\mathrm{Fu}^{1,3}$

$6 \quad{ }^{1}$ School of Kinesiology, Shanghai University of Sport, Shanghai, China

7 2Division of Kinesiology and Health, University of Wyoming, Laramie, United States of

8 America

$9{ }^{3}$ Key Laboratory of Exercise and Health Sciences of Ministry of Education, Shanghai University

10 of Sport, Shanghai, China

12 Corresponding Author:

13 Weijie $\mathrm{Fu}^{1,3}$

14188 Hengren Road, Shanghai, 200438, China

15 Email address: fuweijie@sus.edu.cn; fuweijie315@163.com 
16 Abstract

17 Background Excessive impact peak forces and vertical load rates are associated with running injuries and have been targeted in gait retraining studies. This study aimed to determine the effects of 12-week cadence retraining on impact peak, vertical load rates and lower extremity biomechanics during running.

Methods Twenty-four healthy male recreational runners were randomised into either a 12-week cadence retraining group $(n=12)$, which included those who ran with a $7.5 \%$ increase in preferred cadence, or a control group, which included those who ran without any changes in cadence $(n=12)$. Kinematics and ground reaction forces were recorded simultaneously to quantify impact force variables and lower extremity kinematics and kinetics.

Results Significantly decreased impact peak $(1.86 \pm 0.30 \mathrm{BW}$ vs. $1.67 \pm 0.27 \mathrm{BW}, P=0.003)$, vertical average load rates $(91.59 \pm 18.91 \mathrm{BW} / \mathrm{s}$ vs. $77.31 \pm 15.12 \mathrm{BW} / \mathrm{s}, P=0.001)$ and vertical instantaneous load rates $(108.8 \pm 24.5 \mathrm{BW} / \mathrm{s}$ vs. $92.8 \pm 18.5 \mathrm{BW} / \mathrm{s}, P=0.001)$ were observed in the cadence retraining group, while no significant differences were observed in the control group. Foot angles $\left(18.27^{\circ} \pm 5.59^{\circ}\right.$ vs. $\left.13.74^{\circ} \pm 2.82^{\circ}, P=0.003\right)$ and vertical velocities of the centre of gravity $(\mathrm{CoG})(0.706 \pm 0.115 \mathrm{~m} / \mathrm{s}$ vs. $0.652 \pm 0.091 \mathrm{~m} / \mathrm{s}, P=0.002)$ significantly decreased in the cadence retraining group at initial contact, but not in the control group. In addition, vertical excursions of the $\operatorname{CoG}(0.077 \pm 0.01 \mathrm{~m}$ vs. $0.069 \pm 0.008 \mathrm{~m}, P=0.002)$ and peak knee flexion angles $\left(38.6^{\circ} \pm 5.0^{\circ}\right.$ vs. $\left.36.5^{\circ} \pm 5.5^{\circ}, P<0.001\right)$ significantly decreased whilst lower extremity stiffness significantly increased $(34.34 \pm 7.08 \mathrm{kN} / \mathrm{m}$ vs. $38.61 \pm 6.51 \mathrm{kN} / \mathrm{m}, P=$ 
37 those variables in the control group.

38 Conclusion Twelve-week cadence retraining significantly increased the cadence of the cadence

39 retraining group by $5.7 \%$. This increased cadence effectively reduced impact peak and vertical

40 average/instantaneous load rates. Given the close relationship between impact force variables

41 and running injuries, increasing the cadence as a retraining method may potentially reduce the

42 risk of impact-related running injuries.

43 Keywords: running, cadence, impact peak, lower extremity biomechanics. 


\section{Introduction}

Long-distance running is a very popular form of physical activity in China and across the world (Clermont et al. 2019). According to the Chinese Athletics Association Marathon Annual Press Conference, 5.83 million participants ran in 1,581 marathon events $(5 \mathrm{k}, 10 \mathrm{k}$, half and full marathon) in China in 2018 (Chinese Athletics Association). Similarly, 18.1 million runners registered for organised races in the US (Running USA). However, the incidence of running injuries is fairly high (Messier et al. 2018). 19.4\% to 79.3\% of long-distance runners experienced lower extremity injuries (van Gent et al. 2007). Amongst these injuries, knee injuries, such as patellofemoral pain, are the most common. Meanwhile, there is data to suggest that injuries to the lower leg have been reported to be just as common as injuries to the knee (Buist et al. 2010; Franke et al. 2019).

Training history, anatomical characteristics and running biomechanics are the main risk factors influencing overuse injuries due to running (Hreljac 2004). Amongst various biomechanical factors, excessive impact peak forces and load rates are associated with injuries and have been targeted in gait retraining studies (Cheung \& Davis 2011). In a recent review, excessive accumulation of impact peak forces in knee joints was found to lead to overuse injuries (Gijon-Nogueron \& Fernandez-Villarejo 2015). Previous prospective studies found that injured runners had greater vertical load rates than non-injured runners (Davis et al. 2016; Fu et al. 2017). Another prospective study showed that runners with patellofemoral pain exhibited lower impact loading after the pain and disability typically associated with these injuries were reduced

(Cheung \& Davis 2011). Impact peak can be influenced by several factors, such as speed (Hamill 
et al. 1983), shoe/surface/slope (Dixon et al. 2000; Gottschall \& Kram 2005), strike pattern (Davis et al. 2017) and cadence/step length (Hobara et al. 2012). Increasing running cadence at $2.5 \mathrm{~m} / \mathrm{s}$ or decreasing step length at $4.58 \mathrm{~m} / \mathrm{s}$ could decrease impact peak and vertical load rates (Hobara et al. 2012; Stergiou et al. 2003), and reductions in impact peak were related to decreases in vertical velocity of the centre of gravity (CoG) (Derrick et al. 1998). Other lower extremity variables, such as foot angles at initial contact (Heiderscheit et al. 2011) and peak joint angles during the stance phase (Dos Santos et al. 2016), also showed decreases with increasing cadence. These results indicate that increasing cadence or decreasing step length has an effect on decreasing impact forces and other lower-extremity variables in running.

With regards to cadence retraining, Hafer et al. (2015) observed significant decreases in load rates after 6 weeks of cadence retraining with a $10 \%$ increase in cadence. However, cadence increased by only $2.4 \%$ after retraining, whilst a $10 \%$ increase in cadence was prescribed for retraining; no feedback was given as to how well the participants matched their prescribed cadence during retraining. Whether impact peak would decrease after supervised cadence retraining remains unknown. Therefore, a relatively long-term and supervised intervention is needed to evaluate the effects of cadence retaining on impact peak and load rates. Twelve weeks of gait retraining allows the initial adaptation of musculoskeletal structures to new running patterns (Latorre-Román et al. 2019) and may reduce injury risks of gait transition within a short period (Goss \& Gross 2012). Increasing one's cadence by over 10\% could be metabolically costly, which indicates that considerable increases in cadence are unsustainable and may not be adopted by runners (Cavanagh \& Williams 1982). Mobile monitoring was used by Willy et al. 
86 (2016b) to assess adherence to the prescribed cadence during cadence retraining with a $7.5 \%$

87 increase in cadence, and significant reductions in maximum and average load rates were

88 observed after retraining. As such, we sought to determine whether a relatively small increase in

89 cadence $(7.5 \%)$ during long-term cadence retraining could significantly reduce impact peak and

90 load rates.

91 The present study, therefore, aimed to quantify the effects of a 12-week cadence retraining

92 protocol on impact peak, load rates and other lower-extremity biomechanical variables. We

93 hypothesised that 12-week cadence retraining would result in remarkably decreased impact peak

94 and load rates. Additionally, decreases in lower-extremity biomechanics at initial contact and

95 during the stance phase after cadence retraining would be observed.

97 Methods

\section{Participants}

Sample size estimation indicated that a minimum sample size of 26 participants was required to

achieve a minimum effect size of 0.6 . Considering a drop-out rate of $15 \%-20 \%, 30$ male

recreational runners were recruited through online social media, running clubs and flyers.

Participants were randomly assigned to either a cadence retraining group (CAD) or a control group $(\mathrm{CON})$ on the basis of the lottery method of sampling, and 15 participants were included in each group (Table 1). When the participants first reported to the laboratory, they were required to run on a treadmill for 2 min. A high-speed camera placed next to the treadmill recorded their 
107 108

6

angle (i.e. the angle between the foot and ground at initial contact) of the dominant leg (the preferred kicking leg) (Fu et al. 2017) by reviewing the obtained videos frame by frame (Heiderscheit et al. 2011). Consequently, all runners were rearfoot strikers. They ran a minimum of $15 \mathrm{~km} /$ week for at least 3 months prior to the study. Participants were excluded if they had any lower limb musculoskeletal injuries in the previous 6 months. No significant differences in age, height, weight and weekly mileage were observed between the two groups. This study was approved by the Institutional Review Board of the Shanghai University of Sports (No. 2017007). Informed written consent was obtained from each participant prior to their participation in this study.

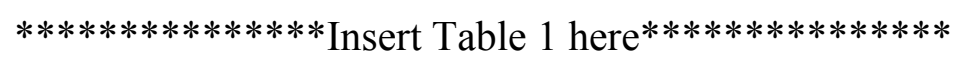

\section{Instrumentation}

A 12-camera motion capture system $(100 \mathrm{~Hz}, \mathrm{~T} 40$; Vicon Motion Inc., Oxford, UK) was used to collect kinematic data. Ground reaction force data were captured by using two $90 \mathrm{~cm} \times 60 \mathrm{~cm} \times$ $10 \mathrm{~cm}$ force platforms $(1,000 \mathrm{~Hz}, 9287 \mathrm{~B}$; Kistler Instruments AG Corp., Winterthur, Switzerland). The kinematics and ground reaction force data were simultaneously collected using the Vicon system. A Photogate system (Witty-Wireless Training Timer, Microgate Corp., Italy) was used to monitor over-ground running speed. Conventional running shoes (Nike Air Zoom Pegasus 34) were used by the participants during the experiments (Fig. 1A). 
128

129

130

131

132

133

134

135

136

137

138

139

140

141

142

143

144

145

146

147

148

\section{Experimental protocol}

The participants visited the laboratory twice, at baseline and at the end of the gait retraining program. Prior to data collection, the participants were required to wear uniform clothing, including a vest, socks and shoes, and walk for $2 \mathrm{~min}$ and run at $3.33 \mathrm{~m} / \mathrm{s}$ on a treadmill for 5 min as a warm-up. Thereafter, a total of 40 markers were placed on the participants, and static calibration was performed. The anatomical locations of the markers were the right/left ilium crest tubercle, right/left posterior superior iliac spine, right/left femur greater trochanter, right/left anterior superior iliac spine, right/left femur lateral epicondyle, right/left femur medial epicondyle, right/left fibula apex of the lateral malleolus, right/left tibia apex of the medial malleolus, right/left head of the fifth metatarsals, right/left head of the first metatarsus and right/left posterior surface of the calcaneus (Fig. 1B). In addition, three tracking markers were placed on the thigh and shank. The participants were instructed to run over the ground across a $10 \mathrm{~m}$ runway (Fig. 1C) at $3.33 \mathrm{~m} / \mathrm{s}$ during which kinematic and ground reaction force data were captured. The running speed was considered acceptable if the deviation was within $5 \%$. Three successful running trials were collected for each participant.

\section{Retraining protocol}

All of the participants were required to run at their preferred speeds during the cadence retraining protocol (Hafer et al. 2015). Running speed and cadence during training were monitored using the commercial running application $\mathrm{CODOON}^{\odot}$ (Chengdu Ledong Information Technology Co., Ltd., China). Each participant received a sport belt bag in which to place their mobile phones 
during running, and they were instructed to place the bag above their sacrum. The participants were asked to run outdoors three times $(30 \mathrm{~min} / \mathrm{run})$ at a comfortable speed to determine their preferred speed and cadence. The preferred speed and preferred cadence were the average values obtained from three outdoor trials. Participants in the CAD group were instructed to run with a $7.5 \%$ increase in cadence, whereas those in the CON group ran without any change in cadence

(Willy et al. 2016b). Participants in the retraining group were informed about and given access to a mobile-based metronome application with tempos set to a $7.5 \%$ increase in cadence. Figure 2 shows the cadence retraining protocol, which lasted for 12 weeks with three sessions a week and

5-48 min each session (Neal et al. 2018). Participants used their preferred running mode,

namely, treadmill or over ground, to complete their retraining. The retraining protocol constituted part of the participants' running volume so that their total weekly running volume remained unchanged. After each retraining session, participants could check their average cadence, speed and running volume on the $\mathrm{CODOON}^{\complement}$ running application. They were also required to submit data recorded by the application to the researchers. Participants were excluded if their training protocols were interrupted more than three times or if their cadence did not achieve the targeted cadence for 3 weeks since the beginning of training. Weekly group trainings were provided three times a week in the CAD group to ensure compliance, and participants chose one of weekly group training sessions in which to participate on the basis of their schedule. During group training, the participants performed an 8 min warm-up, such as dynamic stretching, under the guidance of the researchers. Then, the participants began to run according 
170

171

172

173

174

175

176

177

178

179

180

because the weekly group training aimed to ensure compliance and the quality of retraining.

\section{Data processing}

The Visual 3D software (v5, C-Motion, Inc., Germantown, MD, USA) was used to compute the 3D kinematic and kinetic variables of the lower extremity during running. Marker trajectories were filtered with a cut-off frequency of $7 \mathrm{~Hz}$ via a fourth-order Butterworth low-pass filter (Yang et al. 2020). A seven-segment lower extremity model was built via the Visual 3D, and CoG was estimated from this model. Impact force variables included impact peak, vertical instantaneous load rates (VILR) and vertical average load rates (VALR). In rearfoot strike runners, impact peak was defined the first peak in the ground reaction force curve (Fig. 3B).

Load rates was calculated on the basis of the method described by Futrell et al. (Futrell et al. 2018). In brief, a point of interest (POI) was defined as the first point above $75 \%$ of a participant's body weight with an instantaneous load rate of less than 15 body weight/s. VALR (the average slope) and VILR (i.e. the maximum instantaneous slope) were then calculated from $20 \%$ to $80 \%$ and from $20 \%$ to $100 \%$ of the force at POI, respectively (Fig. 3B). We also calculated lower extremity stiffness (Liu et al. 2006), $k_{\text {leg }}$, as shown in Equation (1). Kinematic variables of the hip, knee and ankle joints included foot angle (the angle between the foot and ground) at initial contact (Fig. 3A) and peak joint extension and peak joint flexion angles during the stance. The times from initial contact to impact peak $\left(t_{i p}\right)$, vertical velocities of the CoG at initial contact and vertical excursion of the CoG during the stance phase were also evaluated. 
191

192

193

194

195

196

197

198

199

200

201

202

203

204

205

206

207

208

209

210

211

$* * * * * * * * * * * * * * *$ Insert Figure 3 here $* * * * * * * * * * * * * * *$

$$
k_{\text {leg }}=\frac{G R F_{i}}{\Delta y}
$$

where $G R F_{i}$ is the vertical ground reaction force at the lowest position of the CoG and $\Delta y$ is the maximum vertical displacement of the CoG.

\section{Statistics}

The mean and standard deviation for each variable was calculated. Two-way repeated measure ANOVA was used to characterise the effects of training (pre- and post-training) and group (CAD and $\mathrm{CON}$ ) on each variable. Independent sample and paired $t$-tests were used as post-hoc tests when a significant interaction was detected to assess potential group effects between CAD and $\mathrm{CON}$ and retraining effects pre- and post-training, respectively. The observed effect size $\left(\eta^{2}\right)$ was considered in the ANOVA results, and effect size (Cohen's $d$ ) was considered in the paired and independent sample $t$-tests results. The $95 \%$ confidence interval (CI) of the differences in group effects was reported. The criterion $\alpha$ level was set to 0.05 . All statistical procedures were conducted using SPSS software (Version 20; SPSS, Inc., Chicago, IL, USA).

\section{Results}

\section{Dropout rate}

Thirty participants (15 in the CAD group and 15 in the CON group) completed the pre-training tests on their first visit to the laboratory (Table 1). However, in the CAD group, one participant was excluded because of insufficient training volume, and two participants withdrew for 
212 personal reasons or because their results showed more than three interruptions. In the CON

213 group, two participants were lost to contact, and one participant withdrew for personal reasons.

214 Overall, 24 participants, 12 in the CAD group and 12 in the CON group, completed the 12-week

215 cadence retraining protocol and reported to the laboratory for post-training tests (Table 1). No

216 significant difference in average running volumes was observed between the CAD and CON

217 groups (CAD: $23.3 \pm 3.3 \mathrm{~km} /$ week, CON: $22.9 \pm 4.3 \mathrm{~km} /$ week).

218

219 Cadence and step length

220 Figure 4A shows a significant training $\times$ group interaction effect for cadence $\left(P<0.001, \eta^{2}=\right.$

221 0.867). Specifically, cadence significantly increased by 5.7\% (161.3 $\pm 9.5 \mathrm{step} / \mathrm{min}$ vs.

222

$170.5 \pm 9.2 \mathrm{step} / \mathrm{min})$ in the CAD group $(P<0.001$, Cohen's $d=3.87)$ but not in the CON group

223

(164.3 $\pm 7.7 \mathrm{step} / \mathrm{min}$ vs. $165.5 \pm 6.8 \mathrm{step} / \mathrm{min}, P>0.05)$ after training. A significant main effect of

training was observed for step length, which decreased by $4.1 \%(2.49 \pm 0.16 \mathrm{~m}$ vs. $2.39 \pm 0.14$

225

$\mathrm{m})$ in the CAD group after training $\left(P=0.011, \eta^{2}=0.259\right)$ (Fig. 4B). No significant difference

for step length was observed in the CON group ( $2.54 \pm 0.16 \mathrm{~m}$ vs. $2.51 \pm 0.14 \mathrm{~m})$. Step length in

the CAD group was $4.9 \%$ lower $(2.39 \pm 0.14 \mathrm{~m}$ vs. $2.51 \pm 0.14 \mathrm{~m})$ than that in the CON group

after training $(P=0.04,95 \% \mathrm{CI}[-0.245,-0.006]$, Cohen's $d=0.94)$.

*****************Insert Figure 4 here

Impact force variables

232 Figure 5A shows significant training $\times$ group interaction effects for impact peak $\left(P=0.022, \eta^{2}=\right.$ 
233 0.217). Impact peak significantly decreased in the CAD group $(P=0.003$, Cohen's $d=1.10)$ but 234 not in the CON group $(P>0.05)$. Meanwhile, impact peak in the CAD group was significantly

235 lower than that in the CON group after training $(P=0.038,95 \%$ CI $[-0.443,-0.013]$, Cohen's $d$ $236=0.95)$. Significant main effects of training were observed for VALR and VILR. Specifically,

$237 \operatorname{VALR}\left(P=0.029, \eta^{2}=0.198\right)$ and $\operatorname{VILR}\left(P=0.025, \eta^{2}=0.209\right)$ decreased in the CAD group 238 after training (Table 2).

\section{Kinematics and joint mechanics}

242 Significant training $\times$ group interaction effects were observed for foot angle $\left(P=0.04, \eta^{2}=\right.$

$0.178)$, vertical velocity of the CoG at initial contact $\left(P=0.035, \eta^{2}=0.186\right)$ and vertical

244 excursion of the $\operatorname{CoG}\left(P=0.001, \eta^{2}=0.409\right)$. Foot angle $(P=0.003$, Cohen's $d=1.09)$, vertical velocity of the CoG at initial contact $(P=0.002$, Cohen's $d=1.16)$ and vertical excursion of the CoG $(P<0.001$, Cohen's $d=1.83)$ decreased in the CAD group after training (Table 2, Fig. 5).

Moreover, vertical excursion of the $\mathrm{CoG}$ in the CAD group was significantly lower than that in the CON group after training $(P=0.025,95 \%$ CI $[-0.015,-0.001]$, Cohen's $d=1.03)$.

Significant main effects of training were observed for peak knee flexion angle. Specifically, peak knee flexion angle $\left(P=0.048, \eta^{2}=0.166\right)$ was decreased in the CAD group after training (Table increased in the CAD group after training $\left(P=0.048, \eta^{2}=0.166\right)$ (Table 2$)$. 


\section{Discussion}

255 This study aimed to characterize the effects of a 12-week cadence retraining protocol on impact

256 peak, load rates and other lower-extremity biomechanical variables. Significant reductions in

257

258

259

260

261

262

263

264

265

266

267

268

269

270

271

272

273

274

impact peak and load rates were observed in the CAD group. The preferred cadence in the CAD group significantly increased after 12-week cadence retraining, consistent with the results of previous studies conducted by Hafer et al. (2015) and Neal et al. (2018). However, the average change in preferred cadence in the present study was $+5.7 \%$ between pre- and post-training. By contrast, the preferred cadence changes in the studies of Hafer et al. (2015) and Neal et al. (2018) were $+2.4 \%$ and $+7.6 \%$, respectively, which were induced by increases of $10 \%$ and $7.5 \%$ in cadence during retraining. Compared to the study by Hafer et al. (2015), the cadence after training in the current study and the study by Neal et al. (2018) was closer to the prescribed cadence, which was likely due to the enhanced supervision in training. In addition, real-time feedback was provided in Neal et al.'s study, which may lead to the differences in cadence improvements between Neal et al.'s and present study.

In the present study, impact peak was significantly reduced by $10.2 \%$ in the CAD group after training, which was greater than the $7.6 \%$ decrease observed (pre-training vs. post-training) in the study of Hobara et al. (2012). Moreover, impact peak in the CAD group after training was $12 \%$ significantly lower than that in the $\mathrm{CON}$ group after training (CAD vs. CON). This decrease may be related to reductions in vertical velocity and vertical excursion of the CoG (Derrick et al. 1998). According to the impulse-momentum principle, impulse is equal to the change in the body's momentum. During running, the momentum exchange between the ground and a portion 
275 of the body when it comes to a full stop causes an impact peak (Addison \& Lieberman 2015). In

276 the present study, the vertical velocity of the CoG at initial contact was significantly decreased in

277 the CAD group after retraining, but no difference in $t_{i p}$ was observed between pre- and post-

278 training. This finding may indicate that the observed decrease in impact peak in the CAD group

279 after retraining may be due to the decreased vertical velocity of the CoG at initial contact after

280 retraining. The foot angle, which reflects the foot strike pattern during running, significantly

281 decreased with increasing cadence (Allen et al. 2016; Heiderscheit et al. 2011). In addition, a

282 lower impact peak has been found in a smaller foot angle at foot contact with a rearfoot strike

283 (Mercer \& Horsch 2015). In the present study, the foot angle in the CAD group significantly

284 decreased by $4.5^{\circ}$ after retraining, which may partially explain the decrease in impact peak in the

285 CAD group after retraining.

286 VALR and VILR in the CAD group were significantly reduced after retraining, consistent

287 with the findings reported by Hafer et al. (2015) and Willy et al. (2016a). Lieberman et al. (2010)

288 found that the load rates was lower in forefoot strikes than that in rearfoot strikes. In the present

289 study, decreased foot angles in the CAD group after retraining slightly altered the strike pattern,

290 which may contribute to reductions in load rates. Injured runners were reported to have higher

291 load rates than non-injured runners in a prospective investigation (Davis et al. 2016). Therefore,

292 the decrease in load rates after retraining indicate that cadence retraining may reduce the risk of

293 running injuries, such as stress fractures.

294 The knee joint was highly sensitive to changes in cadence during the stance phase

295 (Heiderscheit et al. 2011). 5.7\% (9.2 step/min) increase in cadence induced significant changes

Peer] reviewing PDF | (2019:07:39657:3:0:NEW 17 Jul 2020) 
296

297

298

299

300

301

302

303

304

305

306

307

308

309

310

\section{Conclusion}

312 Twelve-week cadence retraining significantly increased runners' cadence by $5.7 \%$. The

in peak knee flexion angle (Dos Santos et al. 2016; Neal et al. 2018). The increase in cadence in the CAD group decreased the peak knee flexion angle and vertical excursion of the CoG; no significant differences were observed for the hip and ankle joint angles between pre- and posttraining. Additionally, lower extremity stiffness significantly increased in the CAD group after training, which may be due to the reduced vertical excursion of the CoG during the stance phase induced by the decrease in peak knee flexion angle.

Some limitations of this study must be considered when interpreting the results. Firstly, all of the participants were male; whether females would show the same effects after 12-week cadence retraining remains unclear. Secondly, the running biomechanics obtained from a limited run-up $(10 \mathrm{~m})$ with a relatively small area $(60 \mathrm{~cm} \times 90 \mathrm{~cm})$ for foot placement may slightly differ from that obtained during outdoor over-ground running. Moreover, long-term retention effects caused by retraining changes were not evaluated in this study. Finally, whether the training effect will maintain when individuals reach fatigue is unknown, and should be considered in future studies. increased cadence effectively decreased a number of impact force variables, namely, impact peak, VALR and VILR. Given the close relationship between impact force variables and running injuries, increasing the cadence as a retraining method may reduce the risk of some impactrelated injuries. A decrease in foot angle at initial contact after training may provide a 
317 mechanical explanation for the observed decrease in impact force variables. Furthermore, the

318 vertical excursion of the CoG decreased, thereby increasing lower extremity stiffness. Hence,

319 cadence retraining can lead to lower extremity biomechanical changes.

320

\section{Funding}

322 This study was supported by the National Natural Science Foundation of China (11772201,

11932013), National Key Research and Development Program of China (2019YFF0302100,

2018YFF0300500), the "Dawn” Program of Shanghai Education Commission (19SG47), Talent

Development Fund of Shanghai Municipal (2018107), Scientific Research Program of Shanghai

Administration of Sports (20Q004), and the High-Level Internationalized Talents Program of

Shanghai University of Sport.

\section{References}

Marathon statistics in China 2019. Available at http://www.athletics.org.cn/marathon/news/2019/0311/218818.html.

Running USA’s Annual Reports 2019 [Internet]. Available at http://www.runningusa.org/annual-reports.

Addison BJ, and Lieberman DE. 2015. Tradeoffs between impact loading rate, vertical impulse and effective mass for walkers and heel strike runners wearing footwear of varying stiffness. J Biomech 48:1318-1324.

Allen DJ, Heisler H, Mooney J, and Kring R. 2016. The Effect Of Step Rate Manipulation on Foot Strike Pattern Of Long Distance Runners. Int J Sports Phys Ther 11:54-63. 
339 Buist I, ., Bredeweg SW, Bessem B, ., Mechelen W, Van, Lemmink KAPM, and Diercks RL. 340 2010. Incidence and risk factors of running-related injuries during preparation for a 4mile recreational running event. Br J Sports Med 44:598.

342 Cavanagh PR, and Williams KR. 1982. The effect of stride length variation on oxygen uptake during distance running. Med Sci Sports Exerc 14:30-35.

344

345

346

Cheung RTH, and Davis IS. 2011. Landing pattern modification to improve patellofemoral pain in runners: a case series. J Orthop Sports Phys Ther 41:914-919.

Clermont CA, Phinyomark A, Osis ST, and Ferber R. 2019. Classification of higher- and lowermileage runners based on running kinematics. J Sport Health Sci 8:249-257.

Davis IS, Bowser BJ, and Mullineaux DR. 2016. Greater vertical impact loading in female runners with medically diagnosed injuries: a prospective investigation. Br J Sports Med $50: 887-892$.

Davis IS, Rice HM, and Wearing SC. 2017. Why forefoot striking in minimal shoes might positively change the course of running injuries. $J$ Sport Health Sci 6:154-161.

Derrick TR, Hamill J, and Caldwell GE. 1998. Energy absorption of impacts during running at various stride lengths. Med Sci Sports Exerc 30:128-135.

Dixon SJ, Collop AC, and Batt ME. 2000. Surface effects on ground reaction forces and lower extremity kinematics in running. Med Sci Sports Exerc 32:1919-1926.

Dos Santos AF, Nakagawa TH, Nakashima GY, Maciel CD, and Serrao F. 2016. The Effects of Forefoot Striking, Increasing Step Rate, and Forward Trunk Lean Running on Trunk and Lower Limb Kinematics and Comfort. Int J Sports Med 37:369-373.

Franke TPC, Backx FJG, and Huisstede BMA. 2019. Running Themselves Into the Ground? Incidence, Prevalence, and Impact of Injury and Illness in Runners Preparing for a Half 
or Full Marathon. J Orthop Sports Phys Ther 49:518-528.

363 Fu W, Fang Y, Gu Y, Huang L, Li L, and Liu Y. 2017. Shoe cushioning reduces impact and muscle activation during landings from unexpected, but not self-initiated, drops. $J$ Sci

366 Med Sport 20:915-920.

Futrell EE, Jamison ST, Tenforde AS, and Davis IS. 2018. Relationships between Habitual Cadence, Footstrike, and Vertical Loadrates in Runners. Med Sci Sports Exerc 50(9):1837-1841.

Gijon-Nogueron G, and Fernandez-Villarejo M. 2015. Risk Factors and Protective Factors for Lower-Extremity Running Injuries A Systematic Review. J Am Podiatr Med Assoc 105:532-540.

Goss DL, and Gross MT. 2012. Relationships among self-reported shoe type, footstrike pattern, and injury incidence. US Army Med Dep J 25-30.

Gottschall JS, and Kram R. 2005. Ground reaction forces during downhill and uphill running. $J$ Biomech 38:445-452.

Hafer JF, Brown AM, Demille P, Hillstrom HJ, and Garber CE. 2015. The effect of a cadence retraining protocol on running biomechanics and efficiency: a pilot study. J Sports Sci $33: 724-731$.

Hamill J, Bates BT, Knutzen KM, and Sawhill JA. 1983. Variations in ground reaction force parameters at different running speeds. Hum Mov Sci 2:47-56.

Heiderscheit BC, Chumanov ES, Michalski MP, Wille CM, and Ryan MB. 2011. Effects of step rate manipulation on joint mechanics during running. Med Sci Sports Exerc 43:296-302.

Hobara H, Sato T, Sakaguchi M, Sato T, and Nakazawa K. 2012. Step frequency and lower extremity loading during running. Int J Sports Med 33:310-313. 
385

386

387

388

389

390

391

392

393

394

395

396

397

398

399

400

401

402

403

404

405

406

407

Hreljac A. 2004. Impact and overuse injuries in runners. Med Sci Sports Exerc 36:845.

Latorre-Román PA, García-Pinillos F, Soto-Hermoso VM, and Muñoz-Jiménez M. 2019. Effects of 12 weeks of barefoot running on foot strike patterns, inversion-eversion and foot rotation in long-distance runners. J Sport Health Sci 8:579-584.

Lieberman DE, Venkadesan M, Werbel WA, Daoud AI, D'Andrea S, Davis IS, Mang'Eni RO, and Pitsiladis Y. 2010. Foot strike patterns and collision forces in habitually barefoot versus shod runners. Nature 463:531-535.

Liu Y, Peng CH, Wei SH, Chi JC, Tsai FR, and Chen JY. 2006. Active leg stiffness and energy stored in the muscles during maximal counter movement jump in the aged. $J$ Electromyogr Kinesiol 16:342-351.

Mercer JA, and Horsch S. 2015. Heel-toe running: A new look at the influence of foot strike pattern on impact force. J Exerc Sci Fit 13:29-34.

Messier SP, Martin DF, Mihalko SL, Ip E, DeVita P, Cannon DW, Love M, Beringer D, Saldana S, Fellin RE, and Seay JF. 2018. A 2-Year Prospective Cohort Study of Overuse Running Injuries: The Runners and Injury Longitudinal Study (TRAILS). Am J Sports Med 46:2211-2221.

Neal BS, Barton CJ, Birn-Jeffrey A, Daley M, and Morrissey D. 2018. The effects \& mechanisms of increasing running step rate: A feasibility study in a mixed-sex group of runners with patellofemoral pain. Phys Ther Sport 32:244-251.

Stergiou N, Bates BT, and Kurz MJ. 2003. Subtalar and knee joint interaction during running at various stride lengths. $J$ Sports Med Phys Fitness 43:319-326.

van Gent RN, Siem D, van Middelkoop M, van Os AG, Bierma-Zeinstra SM, and Koes BW. 2007. Incidence and determinants of lower extremity running injuries in long distance 
runners: a systematic review. Br J Sports Med 41:469-480; discussion 480.

409 Willy RW, Buchenic L, Rogacki K, Ackerman J, Schmidt A, and Willson JD. 2016a. In-field gait retraining and mobile monitoring to address running biomechanics associated with tibial stress fracture. Scand J Med Sci Sports 26:197-205.

412 Willy RW, Meardon SA, Schmidt A, Blaylock NR, Hadding SA, and Willson JD. 2016 b.

413 Changes in tibiofemoral contact forces during running in response to in-field gait $414 \quad$ retraining. J Sports Sci 34:1602-1611.

415 Yang Y, Zhang X, Luo Z, Wang X, Ye D, and Fu W. 2020. Alterations in Running Biomechanics after 12 Week Gait Retraining with Minimalist Shoes. Int J Environ Res Public Health 17:818. 
Table $\mathbf{1}$ (on next page)

Demographics for participants 
1

\begin{tabular}{ccccccc}
\hline & First & Second & & & Weekly \\
Group & visit & visit & $\begin{array}{c}\text { Age } \\
\text { (years) }\end{array}$ & $\begin{array}{c}\text { Height } \\
(\mathrm{cm})\end{array}$ & $\begin{array}{c}\text { Weight } \\
(\mathrm{kg})\end{array}$ & $\begin{array}{c}\text { mileage } \\
(\mathrm{km})\end{array}$ \\
\hline $\begin{array}{c}\text { Cadence } \\
\text { retraining group } \\
\text { Control group }\end{array}$ & 15 & 12 & $23.6 \pm 7.5$ & $174.8 \pm 4.4$ & $71.8 \pm 4.9$ & $23.3 \pm 3.3$ \\
\hline
\end{tabular}

2 
Table 2 (on next page)

Effect of 12-week cadence retraining protocol on lower extremity biomechanics

BW: body weight. 


\begin{tabular}{lcccc}
\hline \multicolumn{1}{c}{ Variables } & \multicolumn{2}{c}{ Cadence retraining group } & \multicolumn{2}{c}{ Control group } \\
& Pre-training & Post-training & Pre-training & Post-training \\
\hline Impact peak (BW) & $1.86 \pm 0.3$ & $1.67 \pm 0.27$ & $1.88 \pm 0.25$ & $1.9 \pm 0.23$ \\
Time from initial contact to impact peak (ms) & $31.29 \pm 1.82$ & $31.74 \pm 2.29$ & $30.13 \pm 4.53$ & $30.6 \pm 4.56$ \\
Vertical average load rates (BW/s) & $91.59 \pm 18.91$ & $77.31 \pm 15.12$ & $92.47 \pm 20.04$ & $91.37 \pm 25.02$ \\
Vertical instantaneous load rates (BW/s) & $108.78 \pm 24.47$ & $92.75 \pm 18.49$ & $109.88 \pm 22.21$ & $107.42 \pm 25.82$ \\
Lower extremity stiffness (KN/m) & $34.34 \pm 7.08$ & $38.61 \pm 6.51^{*}$ & $38.08 \pm 7.35$ & $38.36 \pm 5.59$ \\
Foot angle at initial contact $\left({ }^{\circ}\right)$ & $18.27 \pm 5.59$ & $13.74 \pm 2.82^{*}$ & $17.02 \pm 6.54$ & $16.97 \pm 7.16$ \\
Maximum dorsiflexion angle during stance $\left(^{\circ}\right)$ & $20.10 \pm 4.33$ & $20.50 \pm 3.91$ & $20.63 \pm 3.81$ & $19.74 \pm 4.6$ \\
Maximum knee flexion angle during stance $\left(^{\circ}\right)$ & $-38.60 \pm 5.00$ & $-36.50 \pm 5.45^{*}$ & $-37.74 \pm 2.78$ & $-37.22 \pm 4.42$ \\
Maximum hip flexion angle during stance $\left(^{\circ}\right)$ & $-14.98 \pm 3.27$ & $-14.70 \pm 6.27$ & $-14.39 \pm 4.06$ & $-13.10 \pm 4.41$ \\
\hline
\end{tabular}


Figure 1

(A) Experimental shoes and $(B, C)$ experimental set up 


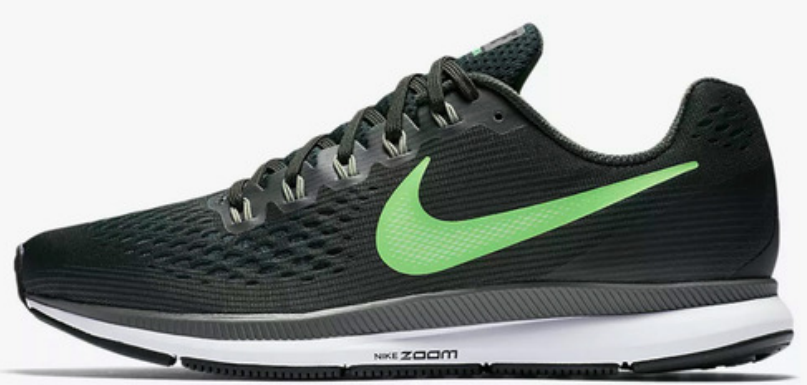

A
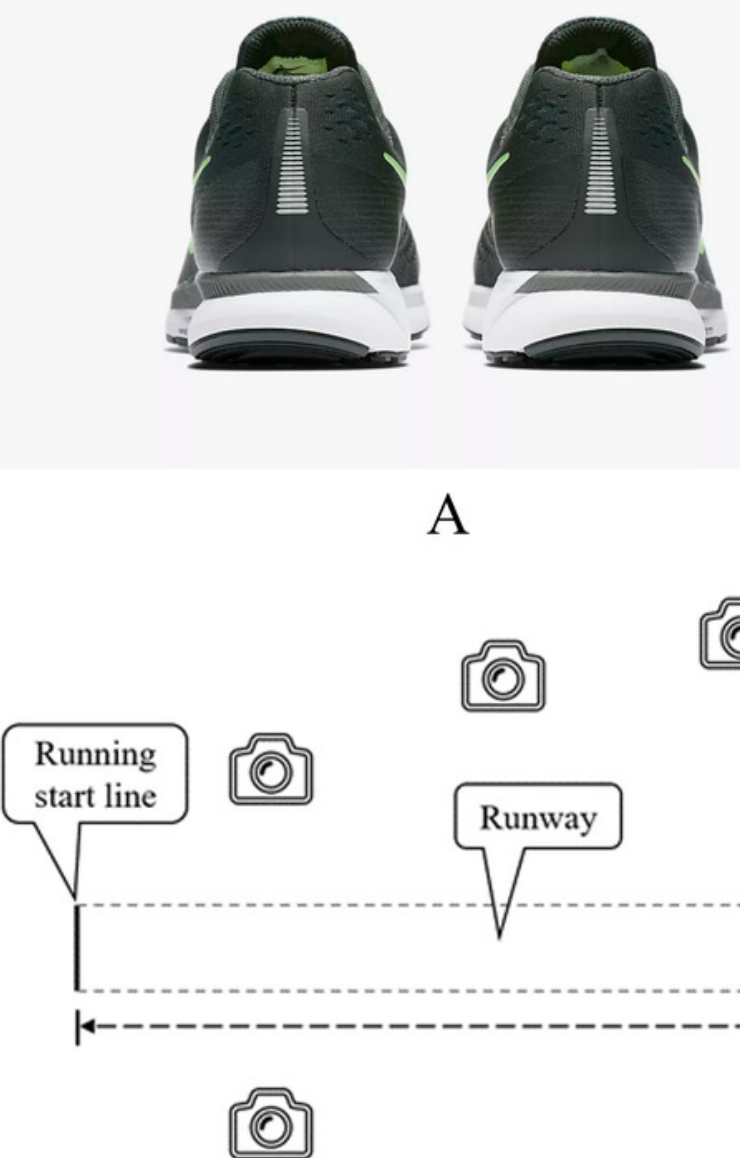

[0]

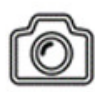

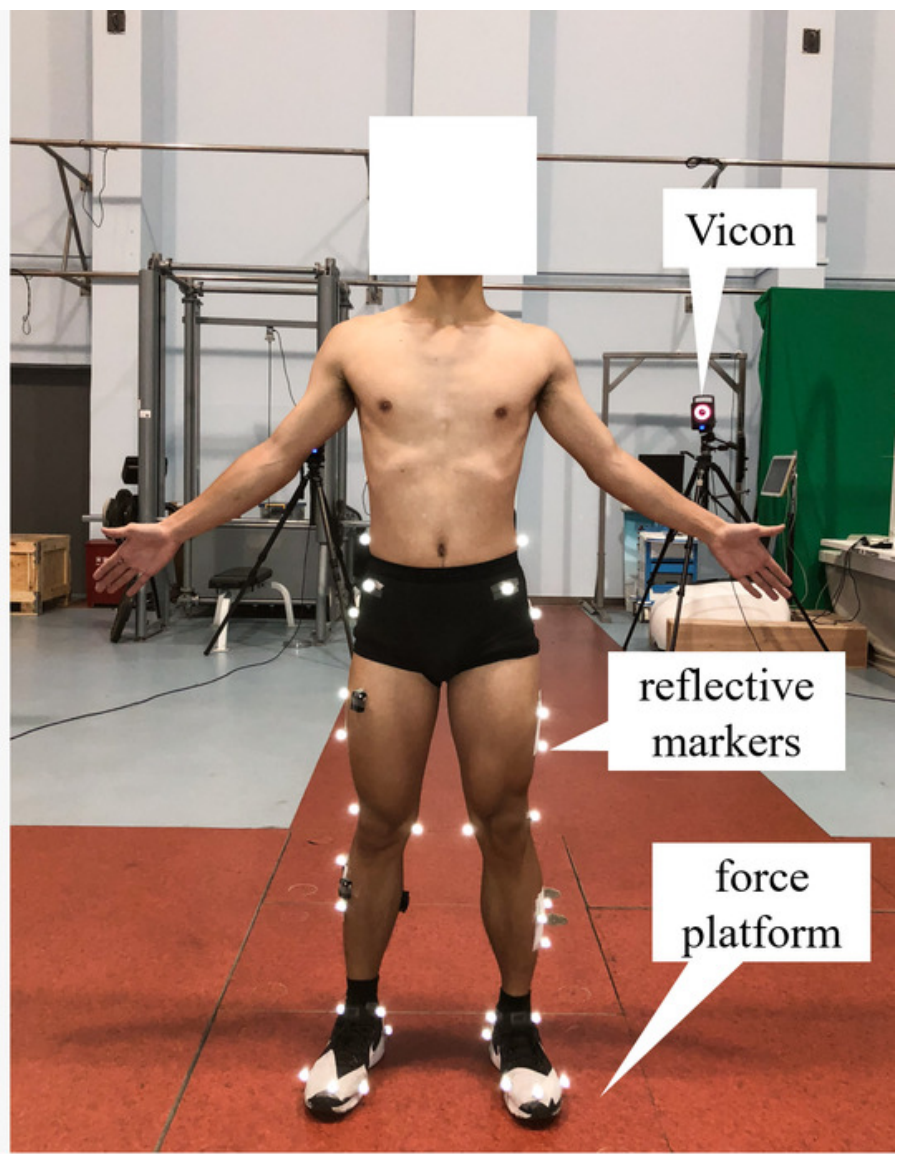

B
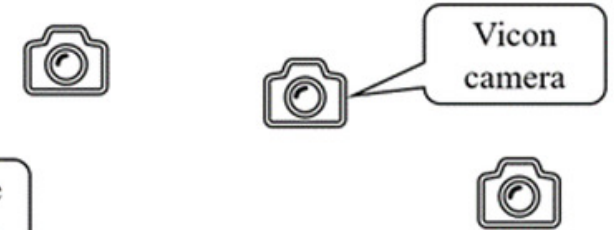

camera
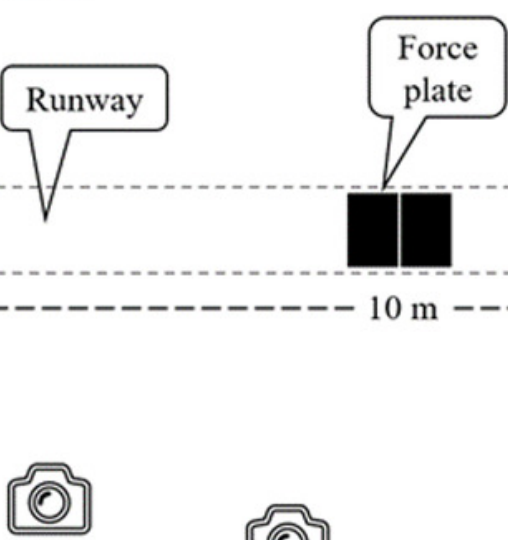

$-10 \mathrm{~m}$

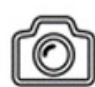

(0)

0

0

\section{C}


Figure 2

Cadence retraining protocol
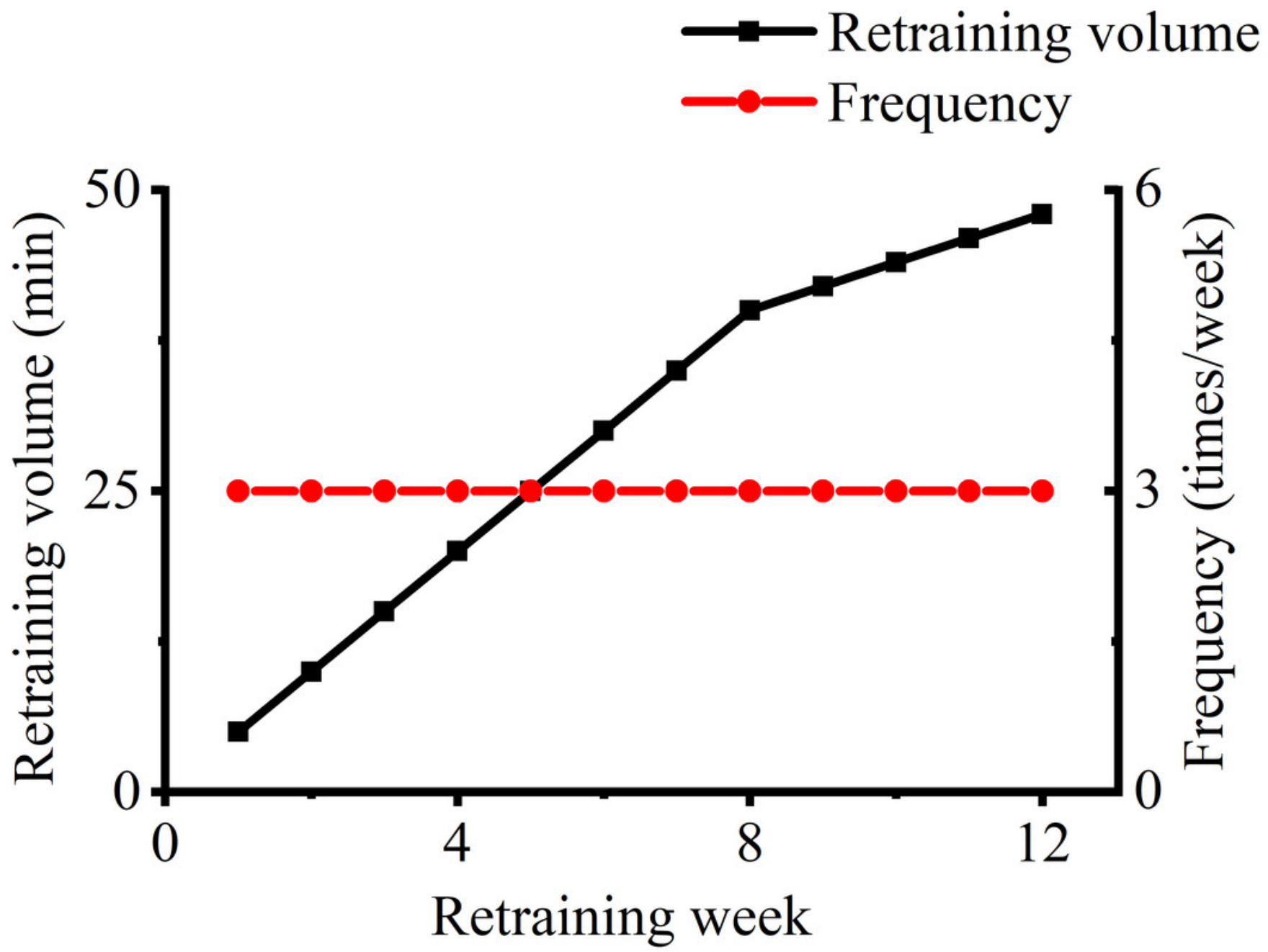
Figure 3

Scheme of (A) lower extremity kinematic and (B) impact force variables

POI, point of interest.

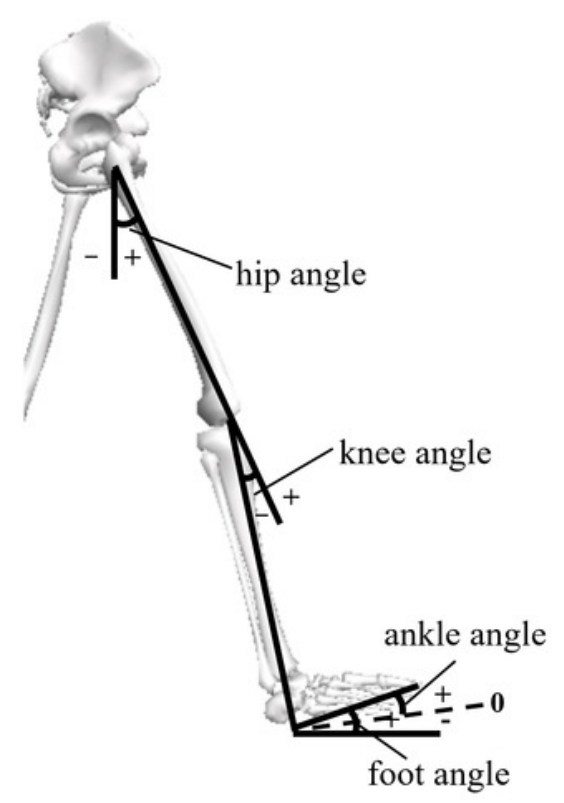

A

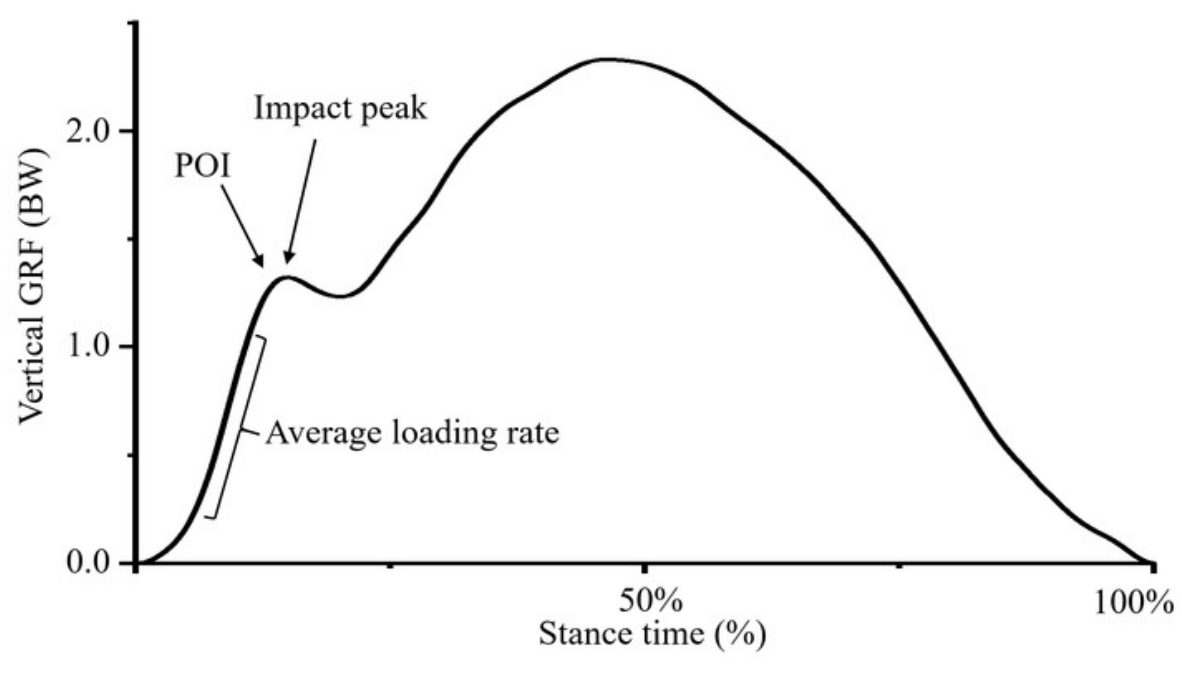

B 
Figure 4

Effect of 12-week cadence retraining protocol on (A) cadence and (B) step length

CAD, cadence retraining group; CON, control group; "indicates significant difference between pre-training and post-training in the CAD; ${ }^{*}$ indicates significant difference between $C A D$ and CON after retraining. $P<0.05$.

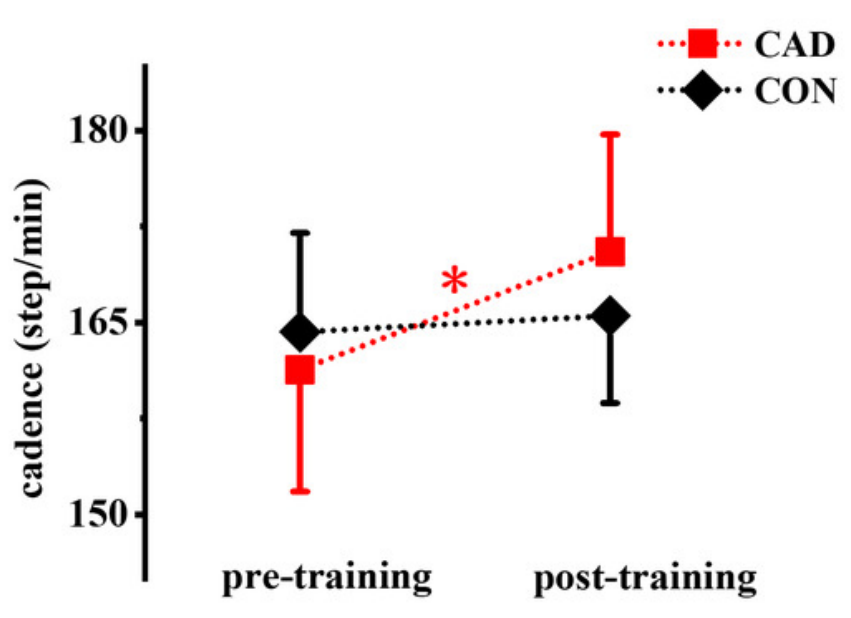

A

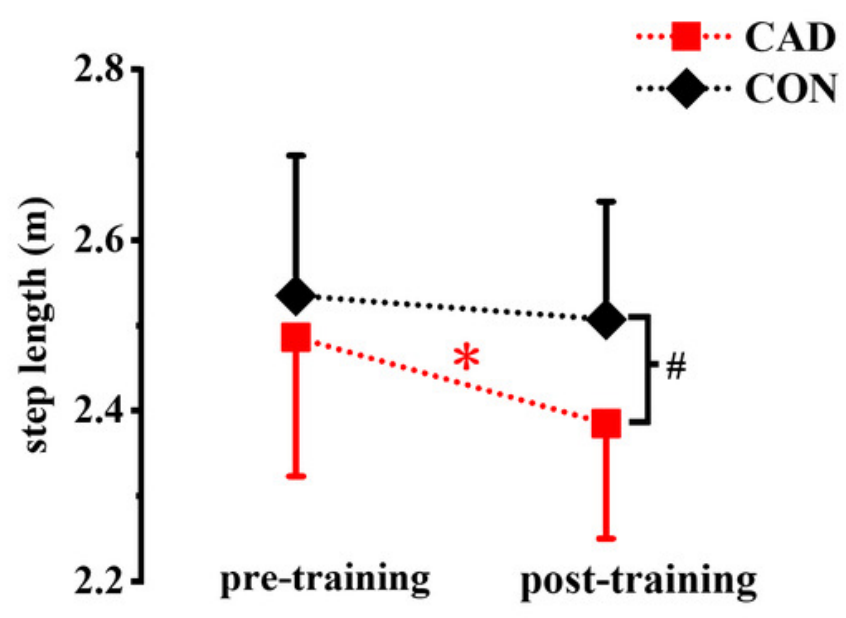

B 
Figure 5

Effect of 12-week cadence retraining protocol on kinematics variables

(A) vertical excursions of the CoG. (B) vertical velocity of the CoG at initial contact. CAD, cadence retraining group; CON, control group; CoG, center of gravity; ${ }^{*}$ indicates significant difference between pre-training and post-training in the CAD; "indicates significant difference between CAD and CON after retraining. $P<0.05$.
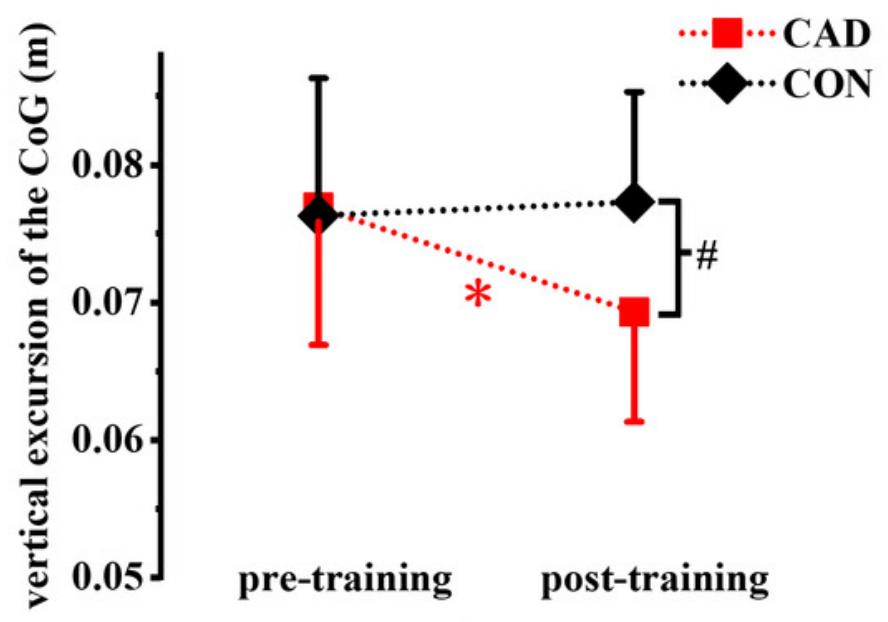

A
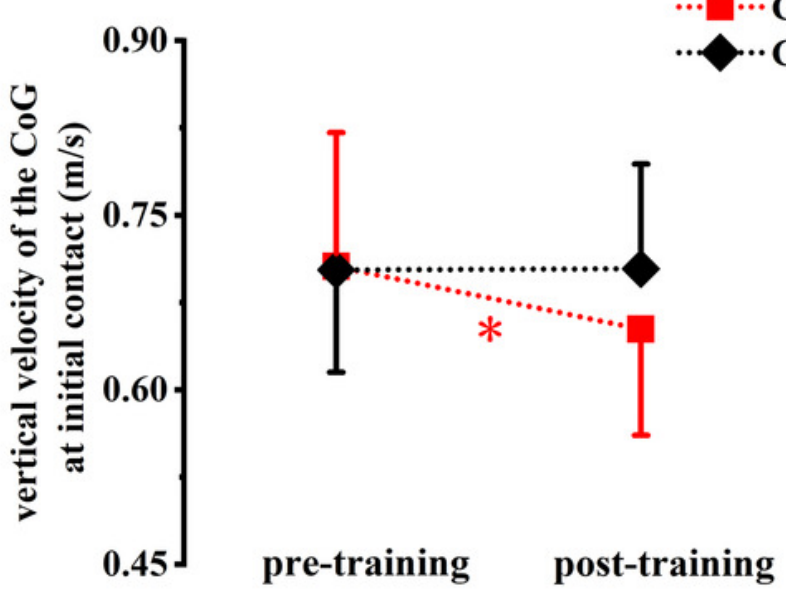

$\mathrm{B}$ 